\title{
Autoeficacia y resiliencia: diferencias entre deportistas practicantes de fitness/culturismo y no deportistas \\ Self-efficacy and resilience: differences between fitness/bodybuilding athletes and non- athletes
}

\author{
María Teresa Ortiz Romero, María E. Garrido Guzmán, Carolina Castañeda Vázquez \\ Universidad de Sevilla (España)
}

\begin{abstract}
Resumen. El estudio de la autoeficacia y la resiliencia favorece en el deportista el desarrollo de la fuerza mental, el control y mejora el rendimiento. El objetivo de esta investigación fue analizar el grado de autoeficacia y el nivel de resiliencia de deportistas de culturismo, fitness y en sujetos no entrenados. Se comprobaron las diferencias en las variables autoeficacia y resiliencia en función del sexo, la práctica deportiva de fitness o culturismo, o la ausencia de ésta. Participaron 179 personas de entre 18 y 69 años (51,4\% hombres y 48,6\% mujeres; $58,1 \%$ culturistas o practicantes de fitness y 41,9\% no hacían ningún tipo de práctica deportiva). Se usó la Escala de Autoeficacia General de Baessler y Schwarzer (1996) para medir la autoeficacia y la Escala de Resiliencia de Connor y Davidson (CD-RISC10) para medir la resiliencia. Los resultados indicaron que, aunque los hombres presentaron un perfil más resiliente que las mujeres, fueron las personas que no realizaban práctica deportiva quienes mayormente reunían dicho rasgo, aunque las diferencias no fueron significativas en este aspecto. Los resultados mostraron mejores percepciones de autoeficacia en culturistas y practicantes de fitness, así como de autoeficacia en los hombres sobre las mujeres. Se demostró la variabilidad de la resiliencia y sus dimensiones en función del deporte practicado, mostrándose los factores resilientes más débiles y que deberían ser desarrollados para mejorar el rendimiento deportivo en situaciones adversas.
\end{abstract}

Palabras clave: entrenamiento, fuerza, salud, deporte, habilidades psicológicas.

\begin{abstract}
The study of self-efficacy and resilience is a challenge for sports psychology, favoring in the athlete the development of mental strength, control and improvement of performance. The objective of this research is to analyze the degree of self-efficacy and the level of resilience of the subjects. It is verified if there are significant differences in these variables, depending on sex, sports practice of fitness or bodybuilding and the absence of practice. This descriptive and cross-sectional study carry out using a sample of 179 people residing in Spain, between 18 and 69 years old (51.4\% are men and 48.6\% women; 58,1\% bodybuilders or fitness people and 41,9\% didn't do any type of sports practice).The Baessler and Schwarzer (1996) General Self-Efficacy Scale is used to measure self-efficacy and the ConnorDavidson Resilience Scale (CD-RISC10) to measure resilience. The results obtained show, according to sex, that men present higher values in resilience and self-efficacy than women and, according to sport activity, that bodybuilders and fitness practitioners present better perceptions of self-efficacy, although not of resilience, than those who don't do any sport. In conclusion, the variability of resilience and its dimensions depending on the sport practiced is demonstrated, showing the weakest resilient factors that should be developed to improve sports performance in adverse situations.
\end{abstract}

Keywords: training, strength, health, sport, psychological skills.

\section{Introducción}

\section{Autoeficacia y resiliencia en la práctica depor- tiva}

Bandura (1986) acuña el término autoeficacia para referirse a «los juicios de cada individuo sobre sus capacidades, con base en las cuales organizará y ejecutará sus actos de modo que le permitan alcanzar el rendimiento deseado» (citado en Guillén, 2007, p.373). La autoeficacia influye en el pensamiento de las personas, permitiendo que ésta atribuya una causa y un significado a una experiencia,

Fecha recepción: 19-04-21. Fecha de aceptación: 27-10-21

María Garrido Guzmán

mariagarrido@us.es siendo esto influyente en los resultados que obtenga el sujeto en la actividad en cuestión.

Fornet (2018) explica que la autoeficacia de un sujeto está moldeada por las referencias que ha tenido a lo largo de su vida. Según qué modelos se hayan tenido y dependiendo de las creencias que se sostengan sobre la inteligencia y la capacidad de aprendizaje, las posibilidades de tener éxito en algo aumentan o disminuyen.

En materia deportiva, la autoeficacia influye a la hora de crear y adherirse al hábito deportivo en diferentes situaciones e independientemente de las circunstancias (García et al., 2020). Es por ello por lo que, según la evidencia teórica y empírica, se reconoce que es una de las variables sociocognitivas que influyen en la práctica deportiva (Lubans, Okely, Morgan, Cotton, Puglisi \& 
Miller, 2012; Majeed, Jabbar \& Jun, 2017; MirzaeiAlavijeh et al., 2018; Zhang, He \& Chen, 2020; García et al., 2020; Fuentes \& González, 2020; Mella, Nazar, Sáez, Bustos, López \& Cobo, 2021). Además, interviene a la hora de desempeñar un esfuerzo, marcarse metas ambiciosas y desafiantes, aspirar más o menos alto, así como para mantener una actitud de compromiso con uno mismo y con la tarea a realizar (Guillén, 2007; Gutiérrez, Gutiérrez \& Ureña, 2013; Jimeno, Peña, Expósito \& Zagalaz, 2009). Este rasgo favorece en el deportista el desarrollo de la fuerza mental, el control y mejora el rendimiento, es decir, cuanto más eficiente se percibe el sujeto, más favorable es su desempeño en la actividad y menor la sensación de estrés y ansiedad.

La autoeficacia es una variable motivacional que podría influir, en gran medida, en los resultados del deportista, constituyendo, por ello, un aspecto clave a trabajar y estudiar (Abalde \& Pino, 2016). Además, en determinadas ocasiones, los juicios que tengan sobre sí mismos los deportistas son mejores predictores del rendimiento deportivo que el nivel de habilidad real de los mismos (Valiante, 2000).

Según estudios revisados, las personas deportistas obtienen mayores puntuaciones en los niveles de autoeficacia que los sujetos sedentarios (Boix, León \& Serrano, 2017; González \& Valadez, 2016; López \& Reyes, 2018; Quesada \& Gómez, 2017), existiendo diferencias según sexo a favor de los hombres mayores deportistas, quienes obtienen mejores puntuaciones que las mujeres mayores deportistas (Reigal \& Videra, 2013).

Junto a lo anterior, diversos estudios indican que la autoeficacia, junto a otros efectos positivos, puede darse en relación con el aumento de los niveles de resiliencia y viceversa, puede influir en la mejora de la resiliencia durante la superación de situaciones adversas (Forés \& Grané, 2008; Ruiz \& López, 2012;Young, 2014; Carbajal $\&$ Delgado, 2020). De este modo, la autoeficacia se enlaza con el concepto de resiliencia. Son muchos los autores que hacen su aportación al término, dándole una forma similar y con matices levemente distintos. En el ámbito de la psicología, se explica que la resiliencia es, según Fornet (2018), «la capacidad de reponerse tras un agente perturbador o un evento estresante» (p.168).

El interés por evaluar la resiliencia nace de que diversos estudios determinan los efectos positivos que tiene el constructo, ya sea gestionando adecuadamente las situaciones de estrés, aumentando la capacidad de logro o mejorando la autoeficacia (Forés \& Grané, 2008; Chacón, Castro, Espejo \& Zurita, 2016). Concretamente, el estudio de la resiliencia como capacidad para su- perar situaciones de adversidad ha tomado protagonismo en los últimos años (Chacón et al., 2016). Además, la actividad física es una herramienta clave para potenciar dicha capacidad en el ser humano (Jerez \& Cabrera, 2021).

Así, en el ámbito deportivo, la literatura evidencia que, a mayor resiliencia, mayor rendimiento en el deporte (Chacón et al., 2016) y bienestar psicológico, más autoconfianza y mejor desempeño, cosa que también sucedía con la autoeficacia. Además, se manifiesta que, altos niveles de resiliencia en personas deportistas se relacionan con mejores estrategias para superar situaciones adversas, así como para gestionar el estrés y responder ante él (Bretón, Zurita \& Cepero, 2016). Al mismo tiempo, es una capacidad que se puede desarrollar (Young, 2014).

En esa línea, según diversos estudios, existe una estrecha relación entre practicar actividades recreativas físicas o, lo que es lo mismo, deporte a conciencia y desarrollar el rasgo de resiliencia (García, Molinero, Ruíz, Salguero, De la Vega \& Márquez, 2014; Gould, Dieffenbach \& Moffett, 2002; Romero, 2015; Romero, 2021). Igual que sucedía cuando se estudiaban las variables sociodemográficas en la autoeficacia, se ponen de manifiesto diferencias según sexo (Zurita et al., 2017).

Además, para promocionar la resiliencia es más efectivo mejorar los factores protectores como la autoeficacia, autoestima, optimismo y/o apoyo social (García et al. 2014). Del mismo modo, Richarson et al. (1990), citado en García et al. 2014, sugieren que las experiencias adversas sirven para potenciar las cualidades resilientes como la autoeficacia, tras una fase de readaptación y lucha contra ese evento identificado por el sujeto como estresante o amenazante.

Asimismo, autores como Chacón et al. (2016), establecen una estrecha relación entre deporte y resiliencia, ya que al desarrollarse esta última, se puede optimizar el rendimiento deportivo. No obstante, existen estudios como el de Serrano, Gómez \& Reche (2021) entre otros (Reche \& Ortín, 2013; Reche, Tutte \& Ortín, 2014; Tutte \& Reche, 2016; Reche, Gómez, Martínez \& Tutte, 2018; Reche, Martínez \& Ortín, 2020), que evidencian lo contrario, afirmando que no siempre el deporte promueve el aumento del rasgo de resiliencia en quienes lo practican. En esos estudios sobre la resiliencia aplicada a gimnastas, judokas, practicantes de hockey y esgrimistas, los autores manifiestan que los deportistas encuestados no presentan elevadas puntuaciones en dicho rasgo, si bien aquellas personas que sí lo hacían demostraban tener mejor rendimiento en la prác- 
tica deportiva que quienes puntuaron bajo o moderado.

En ese sentido, López (2014) concluye que los deportes tradicionales fomentan en menor medida el aumento de los niveles de resiliencia, en comparación con otras modalidades donde la actividad es dirigida o se practica de forma individual.

\section{Culturismo y fitness}

El culturismo se entiende como el proceso de desarrollar y mantener el anabolismo muscular mediante entrenamiento pesado y dieta estricta, teniendo como resultado un físico con gran cantidad de masa magra y poca grasa, con vistas a exponerlo ante un jurado en competición (Arbinaga \& Caracuel, 2003).

De acuerdo con Brown (2003), el culturismo parece centrarse más en vivir intensamente el presente desde un punto de vista materialista, poniendo el foco en el cuerpo supermusculado. Concretamente, autores como Hernández (2008) lo definen como el arte de practicar la cultura física en todas sus formas con el objetivo de alcanzar la salud y mantenerla por el equilibrio físico. Además, las exigencias estéticas y sociales que hoy controlan en gran parte la conducta de la sociedad, así como el levantamiento de pesas como forma de desarrollar o potenciar aptitudes físicas, han llevado a que el culturismo se convierta en una tendencia, a la proliferación de los gimnasios y, con ello, a crear una opción deportiva al alcance de cualquiera (Arbinaga \& Caracuel, 2003; Estrada, Sánchez, Simón \& Casterad, 2020; Veiga, Valcarce \& de la Cámara, 2020; Gómez, López, Pelayo \& Aguirre, 2021); teniendo este hecho repercusión e influencia en otras áreas, como la económica o la laboral; y adquiriendo el centro deportivo significados relacionados con los valores postmaterialistas propios de las sociedades presentes, como son el mantenimiento de la salud y la estética corporal (Torcal, 1989; Chávez, Cortés, Pelayo, Aguirre \& Gómez, 2021).

En esa línea, definiciones recientes de culturismo se alejan del tradicional y simple concepto de práctica física y materialista. En palabras de Sánchez (2019), «es una actividad corporal que se enfrenta a la idea de «límite»» (p.33). Se le atribuyen matices filosóficos (en la medida en que es una forma de expresión e identidad del ser: un modo de estar en un contexto, de comprender el mundo y de reencontrarse con uno mismo) y empiezan a contemplarlo como un ejercicio multidimensional (Martín, 2019; Sánchez, 2019), aclarando que se trata de una actividad deportiva holística, que permite al sujeto transformarse y desarrollarse en términos de cuerpo y mente. En otras palabras, el ejercicio mental que acompaña a la práctica física, supone para el individuo una forma de conocerse, definirse, superar límites y ampliar su abanico de recursos personales.

Una modalidad más reciente que surge de la anterior y que difiere en el objetivo y modo de práctica es el fitness. Esta disciplina se preocupa por el bienestar, la salud integral y el equilibrio, queriendo el practicante estar en forma y encajar con los cánones de belleza actuales sin llegar a la hipermuscularidad (Rodríguez, 2014). Es decir, con un físico «natural» (Martínez, 2016), trabajado con pesos más livianos y de forma más flexible (Taylor, 1993). En otros términos, el fitness es un estilo de vida, una actividad deportiva y sociocultural mediante la que se puede modificar la composición corporal a través de una alimentación saludable y entrenamiento (Nieto, 2018).

Tanto fitness como culturismo coinciden en hacer modificaciones corporales mediante alimentación y entrenamiento, desarrollando un hábito y formando parte de un estilo de vida, proporcionando incluso una identidad cargada de valores al practicante. Dichas modalidades difieren, principalmente, en el foco (competición o equilibrio), en la intensidad y la motivación con que se desarrolla cada una (Nieto, 2018; Veiga et al., 2020).

Por consiguiente, el presente estudio tiene como objetivos analizar el grado de autoeficacia y el nivel de resiliencia de los sujetos, así como determinar si existen diferencias significativas en función del sexo, la práctica deportiva de fitness/culturismo y la ausencia de práctica.

\section{Material y método}

\section{Diseño y participantes}

Se utilizó un diseño descriptivo transversal con fase correlacional, comparativo y cuantitativo para estudiar las variables autoeficacia y resiliencia según sexo y práctica deportiva. Participaron 179 personas de entre 18 y 69 años (51,4\% hombres y 48,6\% mujeres, de los cuales $58,1 \%$ eran deportistas, concretamente culturistas o practicantes de fitness, y el 41,9\% no hacían ningún tipo de práctica deportiva).

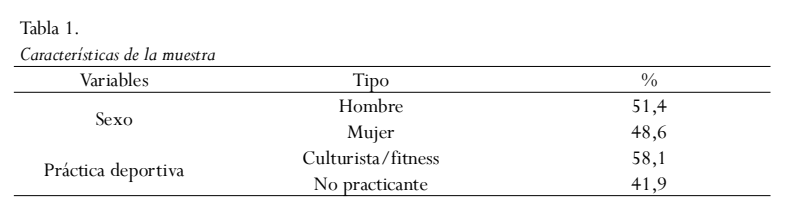

Las características de la muestra pueden observarse en la Tabla 1. En cuanto a la muestra de deportistas se 
recogió que practicaban culturismo/fitness entre uno y siete días semanales, además de que las personas competidoras tenían entre 18 y 51 años $(\mathrm{M}=33,31$; $\mathrm{DT}=$ 13,55). Las mujeres competían en la categoría Bikini Fitness o Body Fitness, mientras que los hombres lo hacían en Men's Physique, Classic Physique y Classic Bodybuilding. Quienes entrenaban por bienestar y salud (física o mental) entraron en el rango de edad de los 20 a los 69 años. El grupo que iba al gimnasio por ocio ocupó de los 20 a los 66 años. Por otro lado, quienes no practicaban deporte tenían edades comprendidas entre los 20 y los 67 años. La muestra fue seleccionada de forma intencional por la facilidad de acceso a los deportistas de los centros deportivos seleccionados (incidental y por conveniencia, para centrar el foco del estudio y los resultados en las variables de interés).

\section{Instrumentos}

Escala de Autoeficacia General de Baessler y Schwarzer (1996). Un test con escalas tipo Likert de 4 puntos, que consta de 10 ítems (e.g., «puedo encontrar la manera de obtener lo que quiero aunque alguien se me oponga»). Su utilidad es medir las expectativas que tienen los sujetos en cuanto a su capacidad para obtener ciertos resultados en el día a día. Su validez (coeficiente alpha de Cronbach $p=0.87)$ se confirma al observar las correlaciones significativas halladas entre los ítems y los constructos con los que se relaciona (adaptación al español en Sanjuán, Pérez \& Bermúdez, 2000).

Escala de Resiliencia de Connor y Davidson (CD-RISC; Connor \& Davidson, 2003). Una escala tipo Likert de 5 puntos con 25 ítems, adaptada al español y reducida a 10 ítems. CD-RISC-10 válida (coeficiente alpha de Cronbach $p=0.9$ ) para medir el nivel de resiliencia (adaptación al español en Crespo Fernández \& Soberón, 2014).

\section{Procedimiento}

El estudio cumplió con las normas éticas del Comité de Investigación y Declaración de Helsinki de 1975 y el procedimiento fue aprobado por el Comité Ético de Experimentación de la Universidad de Sevilla, que tiene como objetivos generales garantizar el respeto a la dignidad, integridad e identidad del ser humano. Se contó en todos los casos con el consentimiento informado de los participantes y se respetó el resguardo a la confidencialidad. Los cuestionarios fueron cumplimentados por personas que encajaron en el perfil descrito, ocupando dicha cumplimentación alrededor de cuatro minutos. Esta fase abarcó desde el 4 de febrero hasta el 18 del mismo mes del año 2020. Siempre hubo un in- vestigador presente durante la recogida de datos para resolver cualquier cuestión que surgiera durante el proceso.

\section{Análisis de datos}

En esta etapa se analiza la información recogida mediante SPSS versión 26. En primer lugar, se usa el coeficiente alpha de Cronbach $(p=0.8$ en ambas escalas) para conocer y verificar la consistencia interna de los instrumentos, y para determinar la normalidad de la distribución se emplea Kolmogorov-Smirnov. Se observa que los datos no siguen una distribución normal $(p<0.5)$. Tras esto, se analizan las propiedades métricas de cada ítem y se determinan los descriptivos básicos (media, desviación típica y porcentajes). La prueba no paramétrica U de Mann-Whitney se emplea con el fin de conocer en qué medida son significativas las diferencias entre las variables y, finalmente, Chi Cuadrado para identificar la asociación estadística significativa.

\section{Resultados}

\section{Percepción de autoeficacia según sexo}

Atendiendo a la información recogida en la Tabla 2, donde se observan los resultados descriptivos y las diferencias entre sexos en relación a la percepción de autoeficacia, se comprueba que más de la mitad de hombres y mujeres participantes se identifican positivamente con las variables estudiadas. Concretamente, se posicionan mayormente en las respuestas «Más bien cierto» $\mathrm{y}$ «Cierto» en las situaciones planteadas en la escala. Si bien es cierto que, el colectivo masculino obtiene medias más elevadas que el femenino en todos los casos, solo en cuatro ocasiones dichas diferencias son estadísticamente significativas, concretamente en los Tabla 2 .

\begin{tabular}{|c|c|c|c|c|c|c|c|c|}
\hline \multicolumn{9}{|c|}{ Chi-Cuadrado Categorías Escala de Autoeficacia } \\
\hline $\begin{array}{l}\text { Ítems Escala de } \\
\text { Autoeficacia }\end{array}$ & Sexo & (a) & $\begin{array}{c}\text { Incorrecto } \\
\%\end{array}$ & $\begin{array}{c}\text { Apenas } \\
\text { cierto } \\
\%\end{array}$ & $\begin{array}{c}\text { Más bien } \\
\text { cierto } \\
\%\end{array}$ & $\begin{array}{c}\text { Cierto } \\
\%\end{array}$ & Total & M DT SIG \\
\hline Obtención & $\begin{array}{c}\text { Hombre } \\
\text { Mujer }\end{array}$ & 0,605 & $\begin{array}{l}3,3 \\
1,1\end{array}$ & $\begin{array}{l}6,5 \\
9,2\end{array}$ & $\begin{array}{l}45,7 \\
50,6\end{array}$ & $\begin{array}{l}44,6 \\
39,1\end{array}$ & 100 & $\begin{array}{llll}3,3 & 0,7 & 0,519 \\
3,2 & 0,6 & 0,519\end{array}$ \\
\hline Resolución & $\begin{array}{c}\text { Hombre } \\
\text { Mujer }\end{array}$ & 0,394 & $\begin{array}{l}1,1 \\
1,1\end{array}$ & $\begin{array}{l}1,1 \\
4,6 \\
\end{array}$ & $\begin{array}{l}37,0 \\
42,5 \\
\end{array}$ & $\begin{array}{l}60,9 \\
51,7\end{array}$ & 100 & $\begin{array}{llll}3,5 & 0,5 & \\
3,4 & 0,6 & 0,171 \\
\end{array}$ \\
\hline Persistencia & $\begin{array}{c}\text { Hombre } \\
\text { Mujer }\end{array}$ & 0,769 & $\begin{array}{l}3,3 \\
2,3 \\
\end{array}$ & $\begin{array}{l}16,3 \\
19,5\end{array}$ & & $\begin{array}{l}43,5 \\
36,8 \\
\end{array}$ & 100 & $\begin{array}{lll}3,2 & 0,8 & \\
3,1 & 0,8 & 0,427 \\
\end{array}$ \\
\hline Confianza & $\begin{array}{c}\text { Hombre } \\
\text { Mujer }\end{array}$ & 0,005 & $\begin{array}{l}1,1 \\
3,4 \\
\end{array}$ & $\begin{array}{c}5,4 \\
21,8 \\
\end{array}$ & $\begin{array}{l}52,2 \\
47,1\end{array}$ & $\begin{array}{l}1,3 \\
27,6 \\
\end{array}$ & 100 & $\begin{array}{lll}3,3 & 0,6 & 0,003 * \\
2,9 & 0,8\end{array}$ \\
\hline Cualidades & $\begin{array}{c}\text { Hombre } \\
\text { Mujer }\end{array}$ & 0,178 & $\begin{array}{l}1,1 \\
1,1\end{array}$ & $\begin{array}{r}4,3 \\
13,8 \\
\end{array}$ & $\begin{array}{l}52,2 \\
47,1\end{array}$ & $\begin{array}{l}42,4 \\
37,9 \\
\end{array}$ & 100 & $\begin{array}{llll}3,3 & 0,6 & \\
3,2 & 0,7 & 0,219 \\
\end{array}$ \\
\hline Tranquilidad & $\begin{array}{c}\text { Hombre } \\
\text { Mujer }\end{array}$ & 0,002 & $\begin{array}{l}1,1 \\
3,4\end{array}$ & $\begin{array}{l}14,1 \\
33,3\end{array}$ & $\begin{array}{l}51,1 \\
48,3\end{array}$ & $\begin{array}{l}33,7 \\
14,9\end{array}$ & 100 & $\begin{array}{lll}3,1 & 0,7 & 0,000 * \\
2,7 & 0,7\end{array}$ \\
\hline Capacidad & $\begin{array}{c}\text { Hombre } \\
\text { Mujer }\end{array}$ & 0,124 & $\begin{array}{l}0,0 \\
3,4 \\
\end{array}$ & $\begin{array}{l}14,1 \\
18,4 \\
\end{array}$ & $\begin{array}{l}54,3 \\
57,5 \\
\end{array}$ & $\begin{array}{l}31,5 \\
20,7 \\
\end{array}$ & 100 & $\begin{array}{ll}3,1 & 0,6 \\
2,9 & 0,7\end{array}$ \\
\hline Esfuerzo & $\begin{array}{c}\text { Hombre } \\
\text { Mujer }\end{array}$ & 0,107 & $\begin{array}{l}0,0 \\
0,0\end{array}$ & $\begin{array}{l}3,3 \\
6,9 \\
\end{array}$ & $\begin{array}{l}38,0 \\
49,4 \\
\end{array}$ & $\begin{array}{l}58,7 \\
43,7 \\
\end{array}$ & 100 & $\begin{array}{lll}3,5 & 0,5 & \\
3,3 & 0,6\end{array}$ \\
\hline Dificultad & $\begin{array}{c}\text { Hombre } \\
\text { Mujer }\end{array}$ & 0,121 & $\begin{array}{l}0,0 \\
2,3 \\
\end{array}$ & $\begin{array}{r}7,6 \\
16,1 \\
\end{array}$ & $\begin{array}{l}48,9 \\
47,1 \\
\end{array}$ & $\begin{array}{l}43,5 \\
34,5 \\
\end{array}$ & 100 & $\begin{array}{llll}3,3 & 0,6 & & \\
3,1 & 0,7 & 0,064 \\
\end{array}$ \\
\hline Alternativas & $\begin{array}{c}\text { Hombre } \\
\text { Mujer }\end{array}$ & 0,558 & $\begin{array}{l}1,1 \\
1,1\end{array}$ & $\begin{array}{l}10,9 \\
18,4\end{array}$ & $\begin{array}{l}46,7 \\
43,7\end{array}$ & $\begin{array}{l}41,3 \\
36,8\end{array}$ & 100 & $\begin{array}{llll}3,2 & 0,7 & \\
3,1 & 0,7 & 0,293 \\
\end{array}$ \\
\hline
\end{tabular}
de Mann-Whitney. *Las diferencias son significativas en el nivel 0,05 (bilateral). 
ítems Confianza (0,003), Tranquilidad (0,000), Capacidad $(0,052)$ y Esfuerzo $(0,036)$.

De este modo, tal y como se refleja en la Tabla 2, el 93,5\% de hombres muestra una confianza elevada («Más bien cierto»y «Cierto») frente al manejo de acontecimientos inesperados, mientras que en el caso de las mujeres el porcentaje es menor (74,7\%). Realizando la prueba no paramétrica U de Mann-Whitney, se observa una diferencia entre medias según sexo estadísticamente significativa $(p=0,003)$, dejando ver que el grupo de hombres siente más confianza en sí mismo $(M=3,3)$ que las mujeres $(M=2,9)$. En la línea de lo anterior, el 84,8\% de hombres y el 63,2\% de mujeres afirman mantener la tranquilidad ante situaciones difíciles por contar con las habilidades necesarias para manejarlas («Más bien cierto»y «Cierto») $(p=0,002)$, presentando también diferencia significativa de medias según U-de Mann-Whitney ( $\mathrm{p} d » 0,000)$ entre hombres $(M=3,1)$ y mujeres $(M=2,7)$. Respecto a la capacidad de manejar cualquier acontecimiento, también se registraron diferencias significativas $(p=0,052)$ en la media entre sexos, siendo superior en el caso de los hombres frente a las mujeres $(3,1$ frente a 2,9 respectivamente). En la misma línea, los hombres $(\mathrm{M}=3,5)$ consideran que pueden resolver la mayoría de los problemas si se esfuerzan lo necesario en mayor medida que las mujeres $(M=3,3)$, siendo estas diferencias significativas según U-de Mann-Whitney $(p=0,036)$.

\section{Percepción de autoeficacia según práctica/no práctica deportiva}

Por su parte, estudiando los porcentajes y las diferencias entre medias según si los participantes practican fitness o culturismo, o en su lugar, no realizan ninguna actividad deportiva, cabe destacar lo siguiente.

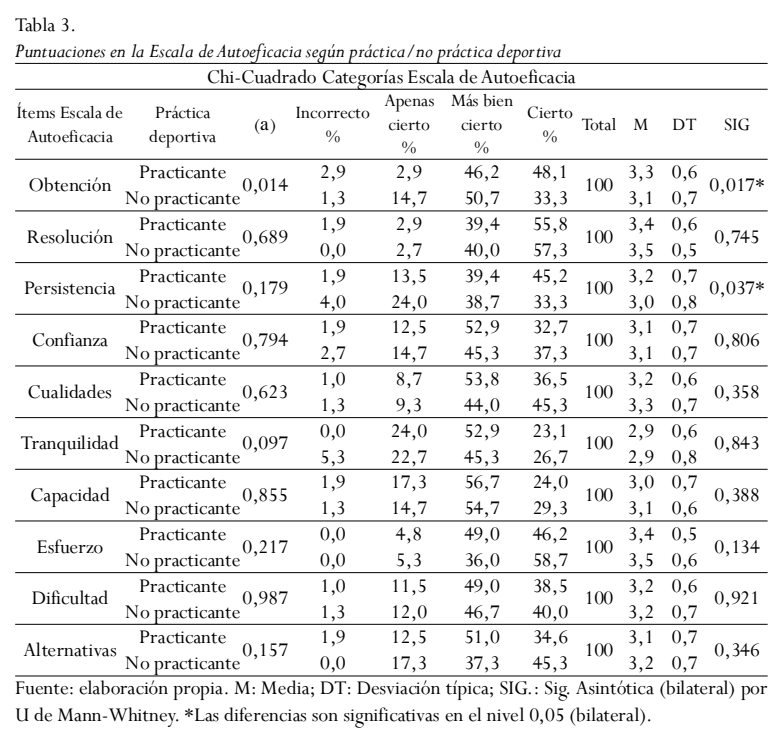

En la Tabla 3, se observa de nuevo que más de la mitad de las personas, sean deportistas o no, se posicionan en los valores más positivos de la escala («Más bien cierto» $\mathrm{y}$ «Cierto»), lo cual muestra que tienen percepciones positivas sobre su autoeficacia. En ocho de los diez ítems, las puntuaciones más positivas corresponden al grupo de personas que practican fitness/culturismo, habiendo diferencias estadísticamente significativas según U de Mann-Whitney en los ítems de Obtención $(p=0,017)$ y Persistencia ( $p=0,037)$. Así, el 94,3\% de culturistas o practicantes de fitness se siente eficaz a la hora de obtener lo que quiere $(M=3,3)$, frente al $84 \%$ de personas que no hacen deporte $(\mathrm{M}=3,1)$; mientras que el $84,6 \%$ de sujetos que practican fitness o culturismo declaran ser persistentes, superando al 72\% que afirma lo mismo perteneciendo al grupo opuesto, dándose aquí la segunda diferencia entre medias estadísticamente significativa $(M=3,2$ frente a $M=3,0$, respectivamente).

\section{Perfil de resiliencia según sexo}

A continuación, se analiza la resiliencia, recogiéndose en la Tabla 4 los datos descriptivos y las diferencias entre medias según sexo.

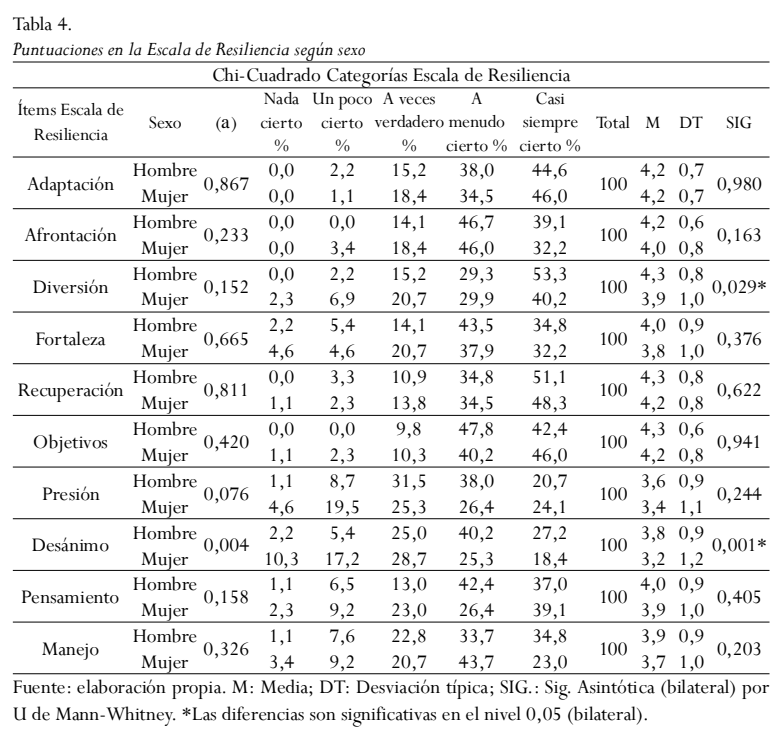

En esta ocasión las respuestas se distribuyen principalmente entre los valores superiores («A menudo cierto» $\mathrm{y}$ «Casi siempre cierto») de la escala, independientemente del sexo, posicionándose más de la mitad de las personas en dichos valores y dándose diferencias significativas en dos de los ítems: Diversión $(p=0,029)$ y Desánimo $(\mathrm{p}=0,001)$.

De este modo, analizando la información del ítem Diversión se observa que el porcentaje de hombres que 
es capaz de ver el lado divertido de las cosas es superior al de las mujeres $(82,6 \%$ de hombres frente al 70,1\% de mujeres), siendo la diferencia de medias significativa $(M=4,3$ y $M=3,9$ respectivamente) según la prueba U de Mann-Whitney. Lo mismo sucede al estudiar el Desánimo sentido por hombres y mujeres $(\mathrm{M}=3,8$ y $\mathrm{M}=3,2)$, pues mientras el $67,4 \%$ de los hombres se posicionan en los valores superiores de la escala («A menudo cierto» $\mathrm{y}$ «Casi siempre cierto»), señalando la afirmación de "No me desanimo fácilmente por los fallos», en el caso de las mujeres suponen 43,7\%, siendo en este caso las medias de los hombres también más elevadas que las mujeres $(M=3,8$ hombres y $M=3,2$ mujeres). En este sentido serían ellos quienes muestran mayor resiliencia que las mujeres al no desanimarse ante los fallos.

\section{Perfil de resiliencia según práctica/no prácti- ca deportiva}

La Tabla 5 muestra los datos del perfil de resiliencia del grupo practicante de fitness/culturismo, frente al colectivo de personas que no hacen actividades deportivas.

\begin{tabular}{|c|c|c|c|c|c|c|c|c|}
\hline \multicolumn{9}{|c|}{$\begin{array}{l}\text { Puntuaciones en la Escala de Resiliencia según práctica/ no práctica deportiva } \\
\text { Chi-Cuadrado Categorías Escala de Resiliencia }\end{array}$} \\
\hline $\begin{array}{c}\text { İtems Escala } \\
\text { de } \\
\text { Resiliencia }\end{array}$ & $\begin{array}{l}\text { Práctica } \\
\text { deportiva }\end{array}$ & $\begin{array}{c}\text { Nada } \\
\text { cierto } \\
\% \\
\end{array}$ & $\begin{array}{l}\text { Un poco } \\
\text { cierto } \%\end{array}$ & $\begin{array}{c}\text { A veces } \\
\text { verdadero } \\
\% \\
\end{array}$ & $\begin{array}{l}\text { A } \\
\text { menudo } \\
\text { cierto \% }\end{array}$ & $\begin{array}{c}\text { Casi } \\
\text { siempre } \\
\text { cierto } \%\end{array}$ & Total & M DT SIG \\
\hline \multirow{2}{*}{ Adaptación } & Practicante & 0,0 & 1,9 & 21,2 & 34,6 & 42,3 & & $\begin{array}{ll}4,1 & 0,8\end{array}$ \\
\hline & No practicante ${ }^{0,305}$ & 0,0 & 1,3 & 10,7 & 38,7 & 49,3 & & $4,3 \quad 0,7^{0,1}$ \\
\hline \multirow{2}{*}{$\begin{array}{c}\text { Afrontació } \\
n\end{array}$} & Practicante & 0,0 & 2,9 & 20,2 & 40,4 & 36,5 & & $4,1 \quad 0,8 \quad 4$ \\
\hline & No practicante 0,087 & 0,0 & 0,0 & $\mathrm{~F} 10,7$ & 54,7 & 34,7 & & $4,2 \quad 0,6^{0}$ \\
\hline \multirow{2}{*}{ Diversión } & Practicante & 0,0 & 5,8 & 19,2 & 32,7 & 42,3 & & $\begin{array}{ll}4,1 & 0,9\end{array}$ \\
\hline & No practicante ${ }^{0,207}$ & 2,7 & 2,7 & 16,0 & 25,3 & 53,3 & & $4,2 \quad 0,9^{0,}$ \\
\hline \multirow[b]{2}{*}{ Fortaleza } & Practicante & 3,8 & 5,8 & 19,2 & 40,4 & 30,8 & & $3,8 \quad 1,0$ \\
\hline & No practicante 0,818 & 2,7 & 4,0 & 14,7 & 41,3 & 37,3 & 100 & $4,0 \quad 0,9^{0,2}$ \\
\hline \multirow{2}{*}{$\begin{array}{c}\text { Recuperaci } \\
\text { ón }\end{array}$} & Practicante & 1,0 & 2,9 & 12,5 & 36,5 & 47,1 & & $\begin{array}{ll}4,2 & 0,8\end{array}$ \\
\hline & No practicante ${ }^{0,855}$ & 0,0 & 2,7 & 12,0 & 32,0 & 53,3 & & $4,3 \quad 0,7^{0,}$ \\
\hline \multirow{2}{*}{ Objetivos } & Practicante & 0,0 & 1,0 & 5,8 & 52,9 & 40,4 & & $\begin{array}{ll}4,3 & 0,6\end{array}$ \\
\hline & No practicante 0,026 & 1,3 & 1,3 & 16,0 & 32,0 & 49,3 & 100 & $4,2 \quad 0,8$ \\
\hline \multirow{2}{*}{ Presión } & Practicante & 2,9 & 13,5 & 27,9 & 32,7 & 23,1 & & $\begin{array}{ll}3,6 & 1,0\end{array}$ \\
\hline & No practicante ${ }^{0,997}$ & 2,7 & 14,7 & 29,3 & 32,0 & 21,3 & & $3,5 \quad 1,0,0,7$ \\
\hline \multirow{2}{*}{ Desánimo } & Practicante & 5,8 & 12,5 & 27,9 & 29,8 & 24,0 & & $3,5 \quad 1,108$ \\
\hline & No practicante 0,837 & 6,7 & 9,3 & 25,3 & 37,3 & 21,3 & 100 & $3,5 \quad 1,1^{0}$ \\
\hline \multirow{2}{*}{$\begin{array}{c}\text { Pensamient } \\
\text { o }\end{array}$} & Practicante & 1,9 & 6,7 & 21,2 & 32,7 & 37,5 & 100 & $3,9 \quad 1,0$ \\
\hline & No practicante ${ }^{0,691}$ & 1,3 & 9,3 & 13,3 & 37,3 & 38,7 & 100 & $\begin{array}{ll}4,0 & 1,0 \\
0,679\end{array}$ \\
\hline \multirow[b]{2}{*}{ Manejo } & Practicante & 1,9 & 11,5 & 25,0 & 32,7 & 28,8 & & $3,7 \quad 1,0$ \\
\hline & No practicante 0,168 & 2,7 & 4,0 & 17,3 & 46,7 & 29,3 & 100 & $3,90,9^{0,187}$ \\
\hline
\end{tabular}

Las respuestas se distribuyen entre los valores centrales y superiores en mayor medida ( $« \mathrm{~A}$ veces verdadero», «A menudo cierto»y «Casi siempre cierto»), independientemente de si las personas hacen deporte o no, sintiéndose identificados más de la mitad de los sujetos con los valores más positivos en todos los ítems de la escala sin que existan diferencias significativas. Al contrario que al estudiar el grado de autoeficacia, en este caso son las personas que no hacen deporte quienes presentan mejores niveles de resiliencia. No obstante, en ninguno de los ítems las diferencias alcanzan a ser significativas.

\section{Discusión}

El presente estudio analiza la autoeficacia y la resiliencia de los sujetos según el sexo y la práctica deportiva de fitness/culturismo.

En el caso de la autoeficacia según el sexo de los sujetos, se ha registrado en la presente investigación que el colectivo masculino obtiene medias más elevadas que el femenino en todos los casos, pero solo en cuatro ocasiones dichas diferencias resultaron estadísticamente significativas: "Tengo confianza en que podría manejar eficazmente acontecimientos inesperados», "Cuando me encuentro en dificultades puedo permanecer tranquilo/a porque cuento con las habilidades necesarias para manejar situaciones difíciles», «Venga lo que venga, por lo general soy capaz de manejarlo» $\mathrm{y}$ «Puedo resolver la mayoría de los problemas si me esfuerzo lo necesario».

Según algunos datos obtenidos en la línea de lo expuesto, el 90,3\% de los hombres frente al 89,1\% de las mujeres sienten que pueden obtener los resultados deseados independientemente de las resistencias externas que se presenten. En cuanto a la resolución de dificultades, el 97,9\% del grupo masculino y el 94,2\% del femenino afirma que, con esfuerzo, en la mayoría de los casos pueden salir adelante. Con relación al alcance de metas, el $80 \%$ de los hombres afirma ser persistente, frente al 78,2\% de las mujeres. Continuando con las cualidades de ambos grupos, un 93,5\% del masculino siente que con sus recursos puede afrontar lo que venga, frente al 85\% del femenino.

En este caso se observa que hay coincidencia con los datos de Reigal y Videra (2013), en la medida en que se encuentran diferencias significativas según sexo, obteniendo mayores puntuaciones en autoeficacia los hombres que las mujeres. En la misma línea, en el estudio de Abalde y Pino (2016), realizado con judokas y deportistas de alto rendimiento, se comprobó que existían diferencias significativas de medias entre hombres y mujeres con respecto a la autoeficacia, registrando que los deportistas varones mantenían niveles más elevados de autopercepción que las mujeres.

De acuerdo con los datos registrados en este estudio acerca de la autoeficacia según la práctica de fitness/ culturismo y la no realización de práctica deportiva, las puntuaciones más positivas corresponden de manera general al grupo de personas que practican fitness/ culturismo. No obstante, las diferencias sólo fueron significativas en dos ítems: «Puedo encontrar la forma de obtener lo que quiero aunque alguien se me oponga» $\mathrm{y}$ «Me es fácil persistir en lo que me he propuesto hasta llegar a alcanzar mis 
metas». Concretamente, el 94,3\% de culturistas o practicantes de fitness se sentía eficaz a la hora de obtener lo que quería, frente al $84 \%$ de personas que no hacían deporte; mientras que el $84,6 \%$ de sujetos que practicaban fitness o culturismo declararon ser persistentes, superando al 72\% que afirmó lo mismo en el grupo de no deportistas.

Estudios revisados (Boix, León \& Serrano, 2017; González \& Valadez, 2016; Reigal, Videra \& Gil, 2014) se mantienen en la misma línea de resultados que los recogidos en la presente investigación. Así, Boix, León y Serrano (2017), en una investigación llevada a cabo entre un grupo de personas que realizaban actividad física y otro que no, observaron que los sujetos inactivos registraron menores puntuaciones de autoeficacia que los grupos activos y que las variables rasgo analizadas no cambiaron significativamente tras seis meses de estudio. Reigal, Videra y Gil (2014), examinaron las relaciones entre práctica física y autoeficacia general, recogiendo que las personas activas tenían mayores niveles de autoeficacia general, así como encontraron diferencias significativas en la percepción de autoeficacia en función de la frecuencia de práctica semanal.

Todo parece indicar una tendencia hacia una percepción de mayor autoeficacia entre las personas activas respecto a las inactivas.

En el caso de la resiliencia, al igual que sucedía con la autoeficacia, los hombres de nuevo muestran unos valores más altos en resiliencia que las mujeres en 9 de los 10 ítems, aunque las diferencias no son significativas más que en los ítems Diversión («Veo el lado divertido de las cosas») y Desánimo («No me desanimo fácilmente por los fallos»). Así, el 82,6\% de hombres era capaz de ver el lado divertido de las cosas frente al 70,1\% de mujeres; y el $67,4 \%$ de los hombres señalaba que no se desanimaba fácilmente por los fallos frente al 43,7\% de las mujeres.

Con relación a las diferencias según sexo, se ha comprobado que estas son significativas en pocos casos, aunque los hombres siguen teniendo medias más elevadas en resiliencia, que las mujeres. Estos datos van en la línea de los recogidos por Zurita et al. (2017), entre otros, quienes sí establecieron diferencias significativas en la resiliencia según el sexo, siendo en dichos casos la percepción de resiliencia superior en el caso de los hombres sobre las mujeres. González-Arratia y Valdez (2013) registraron diferencias significativas entre hombres y mujeres. No obstante, sugieren que las dimensiones de la resiliencia siguen patrones distintos según la edad. En esta misma línea, Gínez-Silva, Morán y Urchaga-Litago
(2019) también por edad y sexo en los adolescentes y en los adultos, siendo en el caso de los adolescentes ellos más resilientes que ellas, mientras que la tendencia se invertía en el caso de los adultos. Por tanto, en la investigación en resiliencia es indispensable continuar profundizando en este tema incluyendo como posible factor modulador la edad de los sujetos, a fin de contar con resultados más consistentes.

En cuanto a la resiliencia según los sujetos que practican fitness/culturismo y aquellos que no realizan ninguna práctica física o deportiva, los datos de la presente investigación han revelado que son las personas que no hacen deporte quienes presentan mejores niveles de resiliencia en los distintos ítems analizados. No obstante, en ninguno de los ítems las diferencias alcanzan a ser significativas entre los dos grupos.

Estos datos contrastan con lo evidenciado en otros estudios revisados (Forés \& Grané, 2008; Ruiz \& López, 2012; Young, 2014), donde se ha comprobado que los niveles de autoeficacia no van alineados con los de resiliencia, pues el grupo que no practica actividades deportivas en este caso puntúa más alto que el de culturistas y practicantes de fitness en la mayoría de los casos, a diferencia de lo escrito por algunos autores (Bretón et al., 2016; Gould et al., 2002).

Es importante aclarar que, tal como se explicó, aunque muchos autores relacionan el deporte con los conceptos de autoeficacia y resiliencia (López \& Reyes, 2018; Gutiérrez et al., 2013; González \& Valadez, 2016; García et al., 2014), no queda claro que exista relación evidente entre hacer deporte y ser más resiliente. Concretamente, el estudio de Castro et al. (2016) reveló que no había diferencias estadísticamente significativas al relacionar la modalidad deportiva practicada (fútbol, balonmano y deportes de invierno) y los niveles de resiliencia. Sin embargo, encontraron un nivel superior de resiliencia en los deportistas profesionales. Asimismo, según los resultados del estudio de Serrano-Nortes, Gómez y Reche (2021), realizado en gimnastas, éstas no presentaban una elevada resiliencia, encontrándose la mayoría dentro de un perfil moderado de resiliencia.

En cualquier caso, se ha de tener en cuenta que el reducido tamaño muestral y la no aleatoriedad del muestreo deben instarnos a tomar estos datos con cautela y continuar con las investigaciones en esta línea para poder observar la tendencia de manera más definida. Sería también interesante, además del fitnessculturismo, entrar a valorar diferentes modalidades deportivas y realizar comparativas más amplias. Otro factor modulador de las variables podría ser la edad, 
por lo que debería considerarse también en análisis posteriores.

\section{Conclusiones}

En relación a la autoeficacia y resiliencia de los participantes en función del sexo, han perfilado una mayor percepción de autoeficacia los hombres frente a las mujeres, especialmente en las variables de Confianza, Tranquilidad, Capacidad y Esfuerzo y las variables Diversión y Desánimo en el caso de la resiliencia.

En líneas generales los resultados esbozan que los hombres tienen percepciones sobre sí mismos más positivas que las mujeres, pues en ambos casos (autoeficacia y resiliencia), los valores obtenidos por el grupo masculino han superado al femenino. De este modo puede afirmarse que sí, existen diferencias de personalidad según sexo a favor de los hombres, llegando a ser en varios casos estadísticamente significativas. Concretamente, ellos tienen más confianza en sí mismos, mantienen la tranquilidad en situaciones adversas en mayor medida y se sienten más capaces de superar tareas si se esfuerzan; de igual forma ven el lado divertido de las cosas con más facilidad y en menor medida se desaniman por los fallos.

En relación a la autoeficacia según la práctica de fitness/culturismo, los deportistas puntuaron más alto de manera general, en ocho de los diez ítems, destacando por significatividad dos de ellos: Obtención y Persistencia. No obstante, en la variable resiliencia no se registraron en ningún caso diferencias significativas.

La tendencia registrada es que las personas deportistas tienen percepciones de autoeficacia más favorables que las pertenecientes al otro colectivo, especialmente a la hora de obtener sus propósitos frente a la adversidad y para persistir en las tareas. Sin embargo, en el caso de la resiliencia no se registran diferencias significativas, aunque parece atisbar una tendencia opuesta, pues quienes no hacen deporte han obtenido mejores valores que las personas que sí lo hacen.

A modo de cierre es necesario resaltar, como se ha explicitado en la discusión, ciertas limitaciones, pues al no trabajar con una muestra muy amplia y ser esta incidental y por conveniencia, así como no incluir más modalidades deportivas ni la edad de los participantes en el análisis, no se extrae información sobre otras características de esta que pudieran incidir sobre las variables analizadas.

Así pues, partiendo de las conclusiones obtenidas, teniendo en cuenta las limitaciones y de cara a futuras investigaciones, se considera pertinente profundizar en el perfil de resiliencia que muestran los practicantes de fitness y culturismo ya que, en contraste con los resultados obtenidos en el presente, en otros estudios (Chacón et al., 2015; Bretón et al., 2016; García et al., 2014; Gould et al., 2002; Romero, 2015) se ha visto que quienes hacen deportes diferentes muestran ser más resilientes que quienes no hacen ninguno. Junto a esto, cabría ahondar en qué exactamente hace que el deporte promueva la percepción positiva de autoeficacia, de modo que, finalmente, se dé respuesta a la pregunta: $¿$ Es el deporte un recurso interesante para promover el desarrollo emocional de las personas?

\section{Referencias}

Abalde, N. \& Pino, R. (2016). Evaluación de la autoeficacia y de la autoestima en el rendimiento deportivo en Judo. Retos:nuevas tendencias en educación fisica, deporte y recreación, (29), 109-113. doi.org/10.47197/retos.v0i29.38461

Arbinaga, F. \& Caracuel, J. C. (2003). Aproximación a la dismorfia muscular. Cuadernos de medicina psicosomática y psiquiatría de enlace, 65(5), 7-15. Recuperado de https:// www.researchgate.net/profile/Felix_Ibarzabal/publication/ 229029807_Aproximacion_a_la_Dismorfia_Muscular/links/ 556436c008ae6f4dcc98ce3a.pdf

Bandura, A. (1986). Social Foundations of Thought and Action.A social cognitive theory. Englewood Cliffs, NJ: Prentice Hall.

Baessler, J. \& Schwarzer, R. (1996). Evaluación de la autoeficacia: Adaptación española de la escala de Autoeficacia General. Ansiedad y Estrés, (2), 1-8.

Boix, S., León, E. \& Serrano, M. (2017). Identificación con el ejercicio físico y autoeficacia: Diferencias entre practicantes de Pilates vs no practicantes. Revista Iberoamericana de Psicología del Ejercicio y el Deporte, 12(1), 99-106. Recuperado dehttps: / /www.redalyc.org/pdf/3111/311148817010.pdf

Bretón, S., Zurita, F. \& Cepero, M. (2016). La resiliencia como factor determinante en el rendimiento deportivo. Revisión bibliográfica. Revista de Ciencias del Deporte, 12(2). Recuperado dehttp://dehesa.unex.es/handle/10662/6784

Brown, D. (2003). El proyecto corporal culturista: construyendo nuevas identidades a través de la supermuscularidad. Ágora para la educación física y el deporte, (2), 61-74. Recuperado de http:/ / uvadoc.uva.es/bitstream/10324/23640/1/AEFD2003-2y3-proyectocorporal-culturista.pdf

Carbajal, M.A. \& Delgado, L.J. (2020). Estrategias de aprendizaje y autoeficacia en alumnos del 1er año de secundaria de los colegios estatales de los balnearios del sur de Lima Tesis de pregrado. Pontificia Universidad Católica del Perú. Recuperado dehttps: / /search.proquest.com/dissertations-theses/estrategias-deaprendizaje-y-autoeficacia-en/docview/2484270762/se2?accountid $=14744$

Castro, M., Chacón, R., Zurita, F., \& Espejo,T. (2016). Niveles de resiliencia en base a modalidad, nivel y lesiones deportivas. 
Retos: nuevas tendencias en educación física, deporte y recreación, 29, $162-165$.

Chacón, R., Castro, M., Espejo,T. \& Zurita, F. (2016). Estudio de la resiliencia en función de la modalidad deportiva: fútbol, balonmano y esquí. Retos: nuevas tendencias en educación física, deporte y recreación, 29, 157-161. doi.org/10.47197/ retos.v0i29.41313

Chávez, O.I., Cortés, P., Pelayo, A.R.,Aguirre, L.E. \& Gómez, L. F. (2021).Tendencias fitness para 2020: Jalisco, México. EmásF: Revista Digital de Educación Física, 68, 131-139. Recuperado de https: / / dialnet.unirioja.es/servlet/articulo?codigo $=7696295$

Connor, K.M. \& Davidson, J.R. (2003). Development of a new resilience scale:The Connor-Davidson resilience scale (CD RISC). Depression and anxiety, 18(2), 76-82. Recuperado de https://onlinelibrary.wiley.com/doi/abs/10.1002/da.10113

Crespo, M., Fernández,V. \& Soberón, C. (2014). Adaptación española de la 'Escala de Resiliencia de Connor-Davidson" (CDRISC) en situaciones de estrés crónico. Behavioral psychology / psicología conductual, 22(2). Recuperado de https:// www.researchgate.net/publication / 260882710_Spanish_Version_of_the_CDRISC_Resilience_Scale_for_Chronic_Stress_Situations

Estrada-Marcén, N., Sánchez-Bermúdez, J., Simón-Grima, J., \& Casterad-Seral, J. (2019). Uso de dispositivos fitness por parte de usuarios de gimnasios (Use of fitness gadgets by gym users). Retos, 38, 26-32. https://doi.org/10.47197/ retos.v38i38.73108

Forés,A. \& Grané, J. (2008). La resiliencia. Crecer desde la adversidad. Barcelona, España: Editorial Plataforma.

Fornet, M. (2018). Feminismoterapéutico. Madrid, España: Editorial Urano.

Fuentes, M., \& González, D. (2020). Adaptación al español del cuestionario de autoeficacia para regular el ejercicio (Spanish adaptation of the self-efficacy questionnaire to regulate exercise). Retos, 38, 595-601. https://doi.org/10.47197/ retos.v38i38.75225

García, F., Herazo,Y., Sánchez, L., Barbosa, E., Coronado, A., Corro, E.,Villarreal,A., \& Redondo, C. (2020). Autoeficacia hacia la actividad física en escolares colombianos (Self-efficacy towards physical activity in Colombian schoolchildren). Retos, 38, 390-395. https://doi.org/10.47197/retos.v38i38.73878

García, X., Molinero, O., Ruíz, R., Salguero, A., De laVega, R. \& Márquez, S. (2014). La resiliencia en el deporte: fundamentos teóricos, instrumentos de evaluación y revisión de la literatura. Cuadernos De Psicología Del Deporte, 14(3), 83-92. Recuperado dehttps://revistas.um.es/cpd/article/view/211291

García, F., Herazo,Y., Sánchez, L., Barbosa, E. J., Coronado,A.del C., Corro, E.A....\& Redondo, C. (2020). Autoeficacia hacia la actividad física en escolares colombianos. Retos: NuevasTendencias En Educación Física, Deportey Recreación, 38, 390-395. doi.org/10.47197/retos.v38i38.7387

Gínez-Silva, Morán y Urchaga-Litago (2019). Resiliencia psicológica a través de la edad y el sexo. International Journal of Developmental and Educational Psychology. 1, monográfico 3, 85-94. DOI: https://doi.org/10.17060/ ijodaep.2019.n1.v4.1513

Gómez-Chávez, L. F. J., López-Haro, J., Pelayo-Zavalza, A., \& Aguirre-Rodríguez,L. (2021). Encuesta Nacional deTendencias Fitness para México en 2021 (National Survey of Fitness Trends in Mexico for 2021). Retos, 42, 453-451. https: / / doi.org/10.47197/retos.v42i0.85947

González-Arratia, N.I. \&Valdez, J.L. (2013). Resiliencia: Diferencias por edad en hombres y mujeres mexicanos. Acta de Investigación Psicológica - Psychological Research Records, (3)1, 941 956.

González, J. \& Valadez,A.(2016). Personalidad y respuesta psicológica en deportistas. Representación temporal y adaptativa del proceso persona-deporte. Retos: nuevas tendencias en educación fisica, deporte y recreación, (30), 211-215. Recuperado dehttps: / /recyt.fecyt.es/index.php/retos/article/view/50267

Gould, D., Dieffenbach, K. \& Moffett,A. (2002). Psychological characteristics and their development in Olympic champions. Journal of Applied Sport Psychology, (14), 172-204.

Guillén, N. (2007). Implicaciones de la Autoeficacia en el rendimiento deportivo. Pensamiento Psicológico, 3(9), 21-32. Recuperado de https://dialnet.unirioja.es/servlet/ articulo?codigo $=2675044$

Gutiérrez, J., Gutiérrez, R. \& Ureña, B. (2013). Autoeficacia general, ansiedad precompetitiva y sensación de fluir en jugadores(as) de balonmano de la selección nacional de Costa Rica. MHSalud: Revista en Ciencias del Movimiento Humano y Salud, 10(2), 1-16. Recuperado de https:// www.revistas.una.ac.cr/index.php/mhsalud/article/view/ $5582 / 5423$

Hernández, C. (2008). Enciclopedia del culturismo. Barcelona: Hispano Europea.

Jerez, P. \& Cabrera-Fernández,A. (2021). Clima motivacional percibido en el deporte y su asociación con los niveles de resiliencia en estudiantes universitarios. Journal of Sport and Health Research, 13(3), 505-514.

Jimeno, R., Peña, P., Expósito,A. \& Zagalaz, M.L. (2009). Elders and physical activity. A simple proposal. Journal of Sport and Health Research, 2(3), 305-328. Recuperado de http:// www.journalshr.com/papers/Vol\%202_N\%203/ V02_3_10.pdf

López, M. (2014). Relación entre satisfacción con la vida y satisfacción con el deporte y en jóvenes deportistas. Tesis Doctoral: Universidad de las Palmas de Gran Canaria.

López, P. \& Reyes, M. (2018). Autoeficacia y Fortaleza Mental en deportistas federados de disciplinas individuales de Lima Metropolitana. Actividad Física y Deporte: Ciencia y Profesión, 28(1), 75-87. Recuperado dehttp:/ / colefcafecv.com/wp-content/ uploads/2018/08/Art5n-28.pdf

Lubans, D., Okely,A., Morgan, P., Cotton,W., Puglisi, L., \& Miller, J. (2012). Description and evaluation of a social cognitive model of physical activity behaviour tailored for adolescent girls. Health Education Research, 27(1), 115-128.

Majeed, N., Jabbar, M., \& Jun, X. (2017). Social cognitive factors associated with physical activity among university students: a cross-sectional study. MOJYoga \& Physical Therapy, 2(2), 50-55. 
Martín, F. (2019). El fisicoculturismo como objeto de análisis antropológico y filosófico. Res Pública: Revista de Historia de Las Ideas Politicas, 22(2), 605-607. https: / / dialnet.unirioja.es/ servlet/articulo?codigo $=7087986$

Martínez,W.A.(2016). Dos o tres cosas que quisiera saber sobre la antropología del fitness. Jangwa Pana, 15(1), 9-12. doi.org/ 10.21676/16574923.1763

Mella, J., Nazar, G., Sáez, F., Bustos, C.E., López,Y. \& Cobo, R. (2021).Variables sociocognitivas y su relación con la actividad física en estudiantes universitarios chilenos. Retos: NuevasTendencias En Educación Física, Deportey Recreación, 40, 76-85. Recuperado de https://dialnet.unirioja.es/servlet/ articulo? codigo $=7698164$

Mirzaei-Alavijeh, M., Soroush, A., Nasirzadeh, M., Hatamzadeh, N., Zinat-Motlagh, F., Jalilian, F., ... Mahboubi, M. (2018). Socio-Cognitive determinants of regular physical activity among college students. World Family Medicine, 16(2), 158-162. doi: 10.5742/MEWFM.2018.93256

Nieto, D.P. (2018). Análisis de la incidencia fitness en la sociedad femenina actual. Tesis Doctoral: Universidad SantoTomás. http:/ /repository.usta.edu.co/handle/11634/12470

Quesada, D. \& Gómez, M. (2017). Perfiles motivacionales de los usuarios de un centro deportivo público. Journal of Sport and Health Research. 9(1), 85-96. Recuperado de http:// www.journalshr.com/papers/Vol\%209_N\%201/ V09_1_8.pdf

Reche, C. \& Ortín, F. (2013). Consistencia de la versión española de la escala de resiliencia en esgrima. Avances de la Psicología del Deporte en Iberoamérica, 2 , 49-57

Reche, C., Tutte,V. \& Ortín, F. J. (2014). Resiliencia, optimismo y burnout en judokas de competición uruguayos. Revista iberoamericana de psicología del ejercicio y el deporte, 9 (2), 271-286.

Reche, C., Gómez, M., Martínez,A. \&Tutte,V.(2018). Eloptimismo como contribución a la resiliencia deportiva. Revista iberoamericana de psicología del ejercicio y el deporte,13(1), 131-136

Reche, C., Martínez-Rodríguez,A., \& Ortín-Montero, F.J. (2020). Characterization of the resilience and exercise dependence athlete. Cultura, Ciencia y Deporte, 15 (43), 17- 26

Reigal, R. \& Videra, A. (2013) Frecuencia de práctica física y autoeficacia en la tercera edad. Revista Internacional de Medicina y Ciencias de la Actividad Física y el Deporte, 13(49), 107-120. Recuperado de https: / repositorio.uam.es/bitstream/ handle/10486/12273/59775_7.pdf?sequence=1

Reigal, R.;Videra,A. \& Gil, J. (2014). Práctica física, autoeficacia general y satisfacción vital en la adolescencia / Physical exercise, general self-efficacy and life satisfaction in adolescence. Revista Internacional de Medicina y Ciencias de la Actividad Física y el Deporte, 14(55), 561-576. Http:/ / cdeporte.rediris.es/revista/revista 55/artdiseno480.htm

Richardson, G.E., Niger, B.L., Jensen, S. \& Kumpfer, K.L. (1990). The resilience model. Health Education, 21, 33-39.

Rodríguez, A. (2014). El fitness es un estilo de vida: Gimnasios y sociabilidad en una perspectiva crítica. VIII Jornadas de Sociología de la UNLP, 3 al 5 de diciembre de 2014, Ensenada,Argentina. EN: Actas. La Plata: UNLP. FAHCE. Departamento de Sociología. Recuperado de http:// w w w. memoria.fah ce.unl p.edu.ar / library? $=\mathrm{d} \& \mathrm{c}=$ eventos\&d $=\mathrm{Jev} 4733$

Romero, C. (2015). Meta-análisis del efecto de la actividad física en el desarrollo de la resiliencia. Retos: nuevas tendencias en educación física, deporte y recreación, 28, 98-103. doi.org/ 10.47197/retos.v0i28.34829

Romero, C.E. (2021). Evaluación de actividades recreativas para el fomento de la resiliencia. Athlos: Revista Internacional de Ciencias Sociales de La Actividad Física, El Juego y El Deporte, 22, 22-44. Recuperado de https: / / dialnet.unirioja.es/servlet/ articulo? codigo $=7767301$

Ruiz, G. \& López,A. (2012). Resiliencia psicológica y dolor crónico. Escritos de Psicología, 5(2), 1-11.

Sánchez,A.J. (2019). Estudio filosófico-estético de la iconografía del cuerpo muscular en la comunicación de masas del siglo XX: el cuerpo hipertrofiado «bodybuilder» como símbolo social y como metáfora politica. Tesis Doctoral: Universidad Complutense de Madrid. https://eprints.ucm.es/id/eprint/51306/

Sanjuán, P., Pérez,A. \& Bermúdez, J. (2000). Escala de autoeficacia general: datos psicométricos de la adaptación para población española. Psicothema, 12(2), 509-513. Recuperado dehttps: / / www.redalyc.org/pdf/727/72797116.pdf

Serrano-Nortes, E., Gómez, M. y Reche, C. (2021). Retos: nuevas tendencias en educación física, deporte y recreación, 41, 581-588.

Taylor, E. (1993). Fitness para mujeres:Plan progresivo de ejercicios con aparatos y pesas ( $1^{\text {a }}$ edición). Madrid, España: Editorial Tutor. S.A.

Torcal, M. (1989). La dimensión materialista / postmaterialista en España: Las variables del cambio cultural. REIS: Revista Española de Investigaciones Sociológicas, 47, 227-254. Recuperado de https: / / dialnet.unirioja.es/servlet/articulo?codigo $=249331$

Tutte,V., \& Reche, C. (2016). Burnout, resiliencia y optimismo en el hockey sobre hierba femenino. Cuadernos de psicología del deporte, 16(3), 73-78.

Valiante, G. (2000).Writing Self-efficacy and Gender Orientation.A developmental Perspective. A Dissertation Proposal. Atlanta, GA: Emory University.

Veiga, O., Valcarce-Torrente, M., \& de la Cámara, M. (2020). Encuesta Nacional de Tendencias de Fitness en España para 2021 (National Survey of Fitness Trends in Spain for 2021). Retos, 39, 780-789. https://doi.org/10.47197/ retos.v1i40.83008

Young, J. (2014). La resiliencia del entrenador. Qué significa, por qué es importante y cómo desarrollarla. ITF Coaching and Sport Science Review, 63(22), 10-12.

Zhang, Z., He, Z., \& Chen,W. (2020). The relationship between physical activity intensity and subjective well-being in college students. Journal of American College Health, 1-6. doi: 10.1080/ 07448481.2020 .1790575

Zurita, F., Zafra, E., Valdivia, P., Rodríguez, S., Castro, M. \& Muros, J. (2017). Análisis de la resiliencia, autoconcepto y motivación en judo según el género. Revista de psicología del deporte, 26(1), 71-81. Recuperado de https: / /ddd.uab.cat/record/ 169756 